\title{
Ckip-1 regulates $\mathrm{C} 3 \mathrm{H} 10 \mathrm{~T} 1 / 2$ mesenchymal cell proliferation and osteogenic differentiation via Lrp5
}

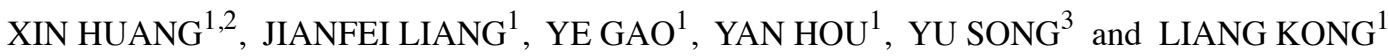 \\ ${ }^{1}$ State Key Laboratory of Military Stomatology and National Clinical Research Center for Oral Diseases and Shaanxi \\ Clinical Research Center for Oral Diseases, Department of Oral and Maxillofacial Surgery, School of Stomatology, \\ The Fourth Military Medical University, Xi'an, Shaanxi 710032; ${ }^{2}$ School of Stomatology of Qingdao University, Qingdao, \\ Shandong 266003; ${ }^{3}$ Department of Orthodontics, Qingdao Stomatological Hospital, Qingdao, Shandong 266001, P.R. China
}

Received October 31, 2019; Accepted September 24, 2020

DOI: $10.3892 /$ etm.2021.9773

\begin{abstract}
Casein kinase-2 interaction protein-1 (Ckip-1) is a negative regulator of bone formation. The identification of novel Ckip-1-related targets and their associated signaling pathways that regulate mesenchymal stem cell (MSC) osteogenic differentiation is required. The present study aimed to evaluate the effects of Ckip-1 knockdown on C3H10T1/2 MSC proliferation and osteogenic differentiation, and to explore the role of the canonical Wnt-signaling receptor Lrp5. Ckip-1-knockdown (shCkip-1), Ckip-1-overexpression (Ckip-1) and their corresponding control [shCtrl and empty vector (EV), respectively] cell groups were used in the present study. Immunofluorescence localization of Ckip-1 was observed. The expression of the key molecules of the canonical Wnt signaling pathway was examined in $\mathrm{C} 3 \mathrm{H} 10 \mathrm{~T} 1 / 2$ cells following osteogenic induction. Moreover, the effects of Lrp5 knockdown in the presence or absence of Ckip-1 knockdown were examined on $\mathrm{C} 3 \mathrm{H} 10 \mathrm{~T} 1 / 2$ cell proliferation and osteogenic differentiation. The results indicated an increase in cell proliferation and osteogenic differentiation in the shCkip-1 group
\end{abstract}

Correspondence to: Professor $\mathrm{Yu}$ Song, Department of Orthodontics, Qingdao Stomatological Hospital, 17 Dexian Road, Qingdao, Shandong 266001, P.R. China

E-mail: songyusy888@163.com

Professor Liang Kong, State Key Laboratory of Military Stomatology and National Clinical Research Center for Oral Diseases and Shaanxi Clinical Research Center for Oral Diseases, Department of Oral and Maxillofacial Surgery, School of Stomatology, The Fourth Military Medical University, 145 West Changle Road, Xi'an, Shaanxi 710032, P.R. China

E-mail: kongliang@fmmu.edu.cn

Abbreviations: Ckip-1, casein kinase-2 interaction protein-1; BTE, bone tissue engineering; MSCs, mesenchymal stem cells; ALP, alkaline phosphatase

Key words: Ckip-1, LDL receptor related protein 5, canonical Wnt signaling pathway, C3H10T1/2 mesenchymal cells, osteogenic differentiation compared with the shCtrl group. The expression levels of LDL receptor related protein 5 (Lrp5), lymphoid enhancer binding factor 1 (Lef1) and transcription factor 1 in C3H10T1/2 cells were significantly increased in shCkip-1 cells following 7-day osteoinduction compared with shCtrl cells. Moreover, the involvement of Lrp5 in shCkip-1-induced osteogenic differentiation of $\mathrm{C} 3 \mathrm{H} 10 \mathrm{~T} 1 / 2$ cells was further verified. The results indicated that Ckip-1 reduced C3H10T1/2 MSC proliferation and osteogenic differentiation via the canonical Wnt-signaling receptor Lrp5, which is essential for the improvement of bone tissue engineering.

\section{Introduction}

Bone defects, which can be caused by trauma, infection, tumors or congenital deformation, have been accepted as difficult-to-treat conditions in medicine (1). To date, autologous bone grafting has been regarded as the most common strategy for the treatment of bone defects (2-4); however, it is considered as a 'wound-repairing-wound' method with disadvantages, such as donor site pain, infection, haemorrhage, nerve damage and limited blood supply (5). The development of cell and molecular biology as well as biomaterial science has enabled the introduction of bone tissue engineering (BTE) as a promising strategy for bone repair (6). During bone repair, the regulation of seed cell osteogenic differentiation is important (7). Mesenchymal stem cells (MSCs) are frequently used for these purposes; therefore, further investigation into the key genes and related signaling pathways of MSCs is required to improve BTE.

Wnt signaling is one of the five main osteogenic signaling pathways (Wnt, bone morphogenic protein (BMP), fibroblast growth factor, Hedgehog and Notch) and includes the three following branches (8): Wnt/ $\beta$-catenin (9), Wnt/peridininchlorophyll-protein complex (10) and $\mathrm{Wnt} / \mathrm{Ca}^{+}(11)$ signaling pathways. Among the different pathways, the Wnt/ $\beta$-catenin (canonical Wnt) signaling pathway is crucial for the maintenance of bone mass (12). Increasing evidence has indicated that the canonical Wnt signaling pathway can repress MSC chondrogenic and adipogenic differentiation (13-15), and improve MSC osteogenic differentiation (15-17). The canonical Wnt signaling pathway can be initiated by the binding of 
Wnt ligands to the receptor complex, which is comprised of Frizzled (FZD) and LDL receptor related protein (Lrp)5/Lrp6, resulting in the accumulation of $\beta$-catenin in the cytoplasm and its translocation to the nucleus to interact with transcription factor 1 (Tcf1)/lymphoid enhancer binding factor 1 (Lef1) (12). Lrp5 is a transmembrane receptor involved in the aforementioned signaling pathway (18). Several recent studies have indicated that inactivation of the canonical Wnt signaling pathway in Lrp5 knockout mice leads to symptoms of bone loss $(19,20)$. By contrast, Lrp5 mutations in mice can result in high bone mass (21).

Casein kinase-2 interaction protein-1 (Ckip-1) mediates interactions with various proteins to participate in different signaling pathways (22). Ckip-1 was initially identified as a negative regulator of the BMP signaling pathway via the activation of Smad Specific E3 Ubiquitin Protein Ligase 1 and the degradation of Smad1/5 (23). However, a limited number of studies have focused on other bone formation signaling pathways. The present study investigated whether Ckip-1 interacted with the canonical Wnt signaling pathway in MSCs, and the role of Lrp5 that may be involved in it.

\section{Materials and methods}

Cell culture and osteogenic induction. C3H10T1/2 cells (donated by the Tissue Engineering Center of the Fourth Military Medical University of China) were cultured at $37^{\circ} \mathrm{C}$ with $5 \% \mathrm{CO}_{2}$ in low glucose DMEM (Gibco; Thermo Fisher Scientific, Inc.) supplemented with $10 \%$ FBS (Gibco; Thermo Fisher Scientific, Inc.) and $0.1 \%$ streptomycin/penicillin. The culture medium was changed every 2 days and the cells were passaged at $\sim 85 \%$ confluence. At $\sim 85 \%$ confluence, osteogenic differentiation was induced by culturing cells in osteogenic medium (DMEM supplemented with 10\% FBS, $10 \mathrm{nM}$ dexamethasone, $10 \mathrm{mM} \beta$-glycerophosphate and $50 \mu \mathrm{g} / \mathrm{ml}$ ascorbic acid; all purchased from Sigma-Aldrich; Merck $\mathrm{KGaA}$ ) for 7 or 14 days at $37^{\circ} \mathrm{C}$ with $5 \% \mathrm{CO}_{2}$.

Cell transfection. C3H10T1/2 cells were seeded ( $1 \times 10^{5}$ cells/well) into 24 -well plates and cultured overnight prior to transfection. Lentivirus-packed Ckip-1 (Ckip-1 overexpression: Ubi-MCS-3FLAG-SV40-EGFP-IRES-puromycin; Ckip-1 knockdown: hU6-MCS-Ubiquitin-EGFP-IRES-puromycin; $1 \times 10^{8} \mathrm{TU} / \mathrm{ml}, 20 \mu \mathrm{l}$; Genechem, Inc.) and Lrp5 knockdown shRNA plasmids (GV248-hU6-MCS-Ubiquiti n-EGFP-IRES-puromycin; 1×10 ${ }^{8} \mathrm{TU} / \mathrm{ml}, 20 \mu \mathrm{l}$; Genechem, Inc.) were transfected into $\mathrm{C} 310 \mathrm{~T} 1 / 2$ cells $\left(1 \times 10^{5}\right.$ cells/well) using polybrene $(50 \mu \mathrm{g} / \mathrm{ml}, 20 \mu \mathrm{l}$; Genechem, Inc.) for $10 \mathrm{~h}$ at $37^{\circ} \mathrm{C}$. The Ckip-1 knockdown shRNA sequences used were as follows: Ckip-1-shRNA1, 5'-CCTGAGTGACTATGAGAA G-3'; Ckip-1-shRNA2, 5'-AGTGCGAAGAGCTCCGGA AA-3'; Lrp5-shRNA1, 5'-GACCTAAAGCGAAUCGAAA-3'; Lrp5-shRNA2, 5'-CGACCTGATGGGACUCAAA-3'; negative control shRNA of shCkip-1, 5'-TTCTCCGAACGTGTC ACGT-3'; and negative control shRNA of shLrp5, 5'-TTCTCC GAACGTGTCACGT-3'. The empty plasmid served as the control of Ckip-1 overexpression group. Following culture for $72 \mathrm{~h}$, fluorescence microscopy and reverse transcription-quantitative PCR (RT-qPCR) were used to verify the results of the transfection.
Immunofluorescence. Cells were seeded into 24-well plates at a density of $70 \%$. Cells were washed three times with PBS and fixed with $4 \%$ paraformaldehyde at $37^{\circ} \mathrm{C}$ for $30 \mathrm{~min}$. Cells were permeabilized for 15 min with $0.2 \%$ Triton X-100 and sealed with 2\% BSA (Sangon Biotech Co., Ltd.) for $30 \mathrm{~min}$ at room temperature. Subsequently, cells were incubated with an anti-Ckip-1 primary antibody (1:200; cat. no. D122120; Sangon Biotech Co., Ltd.) overnight at $4^{\circ} \mathrm{C}$. Following washing with PBS, cells were incubated with a goat anti-rabbit fluorescein-conjugated secondary antibody (1:1,000; cat. no. ab150079; Abcam) for $2 \mathrm{~h}$ in the dark. The nuclei were labeled with DAPI ( $5 \mu \mathrm{g} / \mathrm{ml}$; Sigma-Aldrich; Merck KGaA) for $10 \mathrm{~min}$ at room temperature. Stained cells were visualized using a confocal scanning microscope (magnification, $\mathrm{x} 40$; Nikon Corporation). To observe cell localization, double immunofluorescence staining was performed using rabbit anti-Ckip-1 (1:200; cat. no. D122120; Sangon Biotech Co., Ltd.) and goat anti-Lrp5 (1:1,000; cat. no. ab36121; Abcam) antibodies. Similar to the aforementioned protocol, following fixation, permeabilization and sealing, cells were incubated with anti-Ckip-1 and anti-Lrp5 primary antibodies overnight at $4^{\circ} \mathrm{C}$. Subsequently, cells were incubated with a goat red fluorescein-conjugated secondary antibody $(1: 1,000$; cat. no. ab150079; Abcam) for $2 \mathrm{~h}$ in the dark at room temperature. Following gentle washing with PBS, cells were incubated with a donkey green fluorescein-conjugated secondary antibody (1:1,000; cat. no. ab150129; Abcam) for $2 \mathrm{~h}$ in the dark at room temperature. Following washing with PBS, stained cells were visualized using a confocal laser scanning microscope (magnification, x40; Nikon Corporation).

Proliferation assay. Cell proliferation was analyzed by performing an MTT assay (Sigma-Aldrich; Merck KGaA). Following transfection, C3H10T1/2 cells were seeded ( $2 \times 10^{3}$ cells/well) in 96-well plates and cultured for $24 \mathrm{~h}$. MTT $(10 \mu \mathrm{l})$ was added to each well for $4 \mathrm{~h}$ at $37^{\circ} \mathrm{C}$. Subsequently, the medium was removed and DMSO was added to each well to dissolve the purple formazan. The absorbance was measured at a wavelength of $570 \mathrm{~nm}$ using a Bio-Rad 680 microplate reader (Bio-Rad Laboratories, Inc.).

Alkaline phosphatase (ALP) staining. Cells were seeded $\left(5 \times 10^{5}\right.$ cells/well) in 6 -well plates. Following culture for 14 days, cells were washed with PBS and fixed in $4 \%$ paraformaldehyde at $4^{\circ} \mathrm{C}$ for $30 \mathrm{~min}$. ALP staining was performed using the BCIP/NBT ALP color development kit (LeaGene Biotech Co., Ltd.) according to the manufacturer's protocol. Stained cells were observed using a M205RA stereoscopic light microscope (Leica Microsystems $\mathrm{GmbH}$ ) at a magnification of $\mathrm{x} 200$, and quantified using Image Pro Plus software (version 7.1; Media Cybernetics, Inc.).

$R T$ - $q P C R$. Cells were seeded $\left(5 \times 10^{5}\right.$ cells/well) into 6 -well plates and cultured in DMEM. Subsequently, the medium was removed and cells were washed three times with PBS. Total RNA was extracted using RNAisoPlus (Takara Bio, Inc.) and reverse transcribed into cDNA using Prime Script RT Master Mix (Takara Bio, Inc.) in a 20- $\mu$ l volume. Subsequently, qPCR was performed using a SYBR PCR Master Mix kit (Takara Bio, Inc.) with $10 \mu \mathrm{M}$ specific primers in a 
$25 \mu 1$ total reaction volume with the following thermocycling conditions: Initial denaturation step at $95^{\circ} \mathrm{C}$ for $1 \mathrm{~min}$; followed by 35 cycles at $95^{\circ} \mathrm{C}$ for $30 \mathrm{sec}, 58^{\circ} \mathrm{C}$ for $30 \mathrm{sec}$; and a final extension step at $72^{\circ} \mathrm{C}$ for $30 \mathrm{sec}$. All signals were normalized to GAPDH, and the $2^{-\Delta \Delta \mathrm{Cq}}$ method was used for quantification (24). The mRNA expression levels of Ckip-1, RUNX family transcription factor 2 (Runx2), Osterix (Osx), type I collagen (Col1), bone sialoprotein (Bsp), osteocalcin (Ocn), Lrp5, Lef1 and Tcf1 were assessed via qPCR. The sequences of the primers used are presented in Table I. mRNA expression levels were normalized to the internal reference gene GAPDH.

Western blotting. Total protein was extracted from cells using RIPA lysis buffer (Sangon Biotech Co., Ltd.) and quantified using a BCA protein kit (Sangon Biotech Co., Ltd.). Equal amounts of protein $(20 \mu \mathrm{g} /$ lane $)$ were separated via $10 \%$ SDS-PAGE and transferred onto PVDF membranes, which were blocked in TBST $(0.1 \%$ Tween-20) with $5 \%$ non-fat milk for $1 \mathrm{~h}$ at room temperature. Subsequently, the membranes were incubated with the following primary antibodies overnight at $4^{\circ} \mathrm{C}$ : anti-Ckip-1 (1:500; cat. no. D122120; Sangon Biotech Co., Ltd.), anti- $\beta$-actin (1:3,000; cat. no. 4970; Cell Signaling Technology, Inc.), anti-Lrp5 (1:1,000; cat. no. ab38311; Abcam), anti- $\beta$-catenin (1:1,000; cat. no. 8480; Cell Signaling Technology, Inc.) and anti- $\alpha$-tubulin (1:2,000; cat. no. 2125; Cell Signaling Technology, Inc.). Following primary incubation, the membranes were incubated with a fluorescein-conjugated secondary antibody (1:3,000; cat. no. ab150079; Abcam) for $2 \mathrm{~h}$ at room temperature. Protein bands were visualized using ECL reagent (Sangon Biotech Co., Ltd.) and Odyssey V3.0 image scanning (LI-COR Biosciences).

Statistical analysis. Statistical analyses were performed using SPSS software (version 19.0; SPSS, Inc.). Data are presented as the mean \pm SD from at least three independent experiments. Comparisons among groups were analyzed using one-way ANOVA followed by Tukey's post hoc test. $\mathrm{P}<0.05$ was considered to indicate a statistically significant difference.

\section{Results}

Ckip-1 knockdown and overexpression in C3H10T1/2 cells. Following transfection of $\mathrm{C} 3 \mathrm{H} 10 \mathrm{~T} 1 / 2$ cells with different lentiviral expression vectors and subsequent puromycin selection, transfection efficiency was determined. The majority of cells displayed GFP expression and transfection efficiency was estimated to be $\geq 90 \%$ (Fig. 1A). In addition, the expression levels of Ckip-1 in different groups were measured via RT-qPCR and western blotting. Ckip-1 mRNA (Fig. 1B) and protein (Fig. 1C) expression levels were markedly decreased in the shCkip-1 group compared with the shCtrl group, whereas the opposite results were observed in the Ckip-1 group compared with the EV group, demonstrating successful and effective Ckip-1 knockdown and overexpression, respectively. To further detect the expression of Ckip-1 in C3H10T1/2 cells, immunofluorescence analysis was conducted. The majority of cells with green fluorescence in the shCtrl and EV groups displayed merged signals with red fluorescence that were representative of Ckip-1 detection (Fig. 1D). Decreased expression levels of Ckip-1 were observed in the shCkip-1 group compared
Table I. Primers used for reverse transcription-quantitative PCR.

\begin{tabular}{ll} 
Gene & \multicolumn{1}{c}{ Sequence $\left(5^{\prime} \rightarrow 3^{\prime}\right)$} \\
\hline GAPDH & F: AGGTCGGTGTGAACGGATTTG \\
& R: GTAGACCATGTAGTTGAGGTCA \\
Ckip-1 & F: AACCGCTATGTGGTGCTGAA \\
& R: CAGGGTGAACTTGCTGTGATTT \\
Runx2 & F: GACTGTGGTTACCGTCATGGC \\
& R: ACTTGGTTTTCATAACAGCGGA \\
Osx & F: CCCAGCCACCTTTACCTACA \\
& R: TATGGAGTGCTGCTGGTCTG \\
Col-1 & F: GAGGCATAAAGGGTCATCGTGG \\
& R: CATTAGGCGCAGGAAGGTCAG \\
Bsp & F: GAGCCAGGACTGCCGAAAGGAA \\
& R: CCGTTGTCTCCTCCGCTGCTGC \\
Ocn & F: CAGCTTGGTGCACACCTAGC \\
& R: AGGGTTAAGCTCACACTGCTCC \\
Lrp5 & F: CTGCCAGGATCGCTCTGATG \\
Lef1 & R: ACACTGTTGCTTGATGAGGACACAC \\
& F: GCCACCGATGAGATGATCCC \\
Ref1 & F: TTGATGTCGGCTAAGTCGCC \\
& R: CTGGGCCAACTTCACATCCC
\end{tabular}

Ckip1, casein kinase-2 interaction protein-1; Runx2, RUNX family transcription factor 2; Osx, Osterix; Col-1, type I collagen; Bsp, bone sialoprotein; Ocn, osteocalcin; Lrp5, LDL receptor related protein 5; Lef1, lymphoid enhancer binding factor 1; Tcf1, transcription factor 1 .

with the shCtrl group, whereas increased expression levels of Ckip-1 were observed in the Ckip-1 group compared with the EV group.

Effects of Ckip-1 on C3H1OT1/2 cell proliferation. To determine the effects of Ckip-1 knockdown and overexpression on C3H10T1/2 cell proliferation, an MTT assay was performed. On days 1, 3, 5 and 7, cell proliferation in the shCkip-1 group was significantly increased compared with the shCtrl group. By contrast, on day 5 and 7, cell proliferation in the Ckip-1 group was significantly decreased compared with the EV group (Fig. 2A).

Effects of Ckip-1 on C3H10T1/2 cell osteogenic differentiation. An ALP activity assay and RT-qPCR were performed to evaluate C3H10T1/2 cell osteogenic differentiation. Following 14-day osteoinduction, the shCkip-1 group displayed significantly higher positive staining (dark purple) compared with the shCtrl group (Fig. 2B). By contrast, the Ckip-1 group displayed significantly decreased ALP staining compared with the EV group. Moreover, gene expression analysis of osteogenic markers (Runx2, Osx, Col1, Ocn and Bsp) in the shCkip-1 group demonstrated a significant increase in expression levels compared with the shCtrl group (Fig. 2C). By contrast, with the exception of Bsp, the expression levels of the markers in the Ckip-1 group were significantly decreased compared with the EV group. 

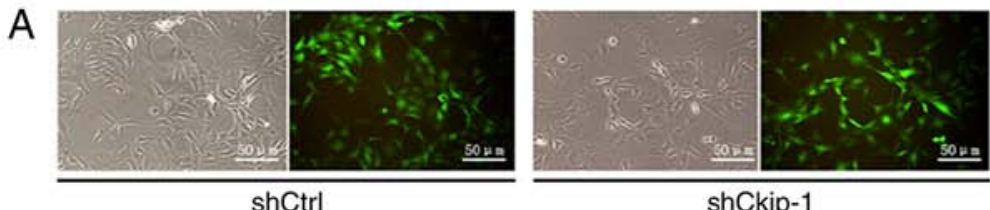

shCkip-1

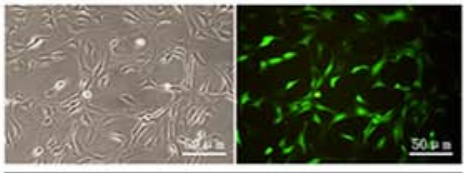

EV

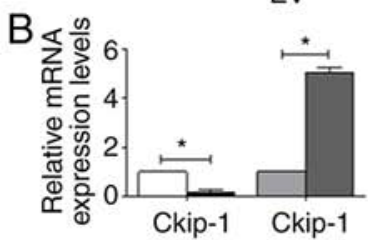

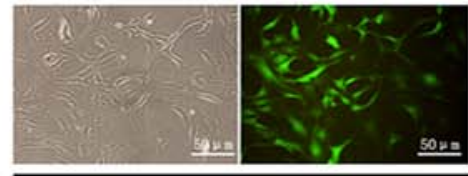

Ckip-1

C

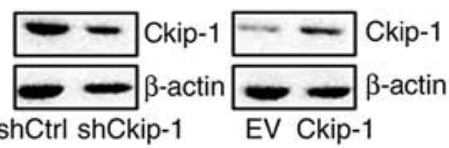

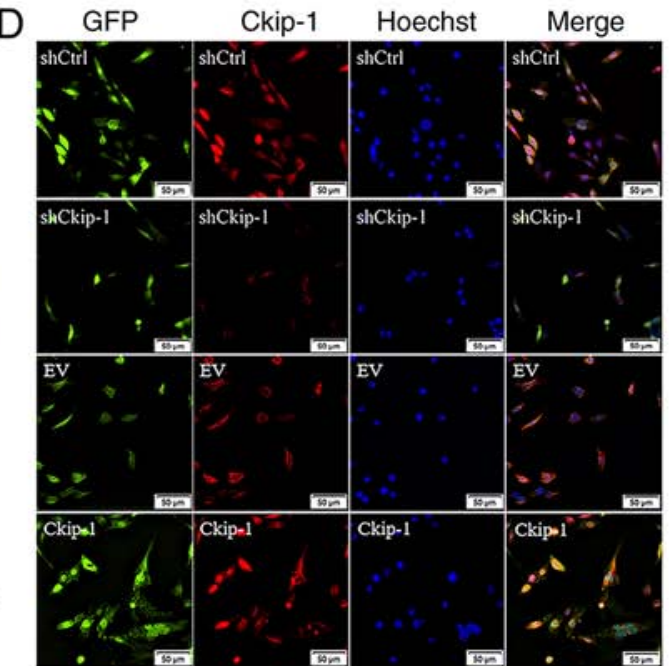

Figure 1. Ckip-1 knockdown and overexpression in C3H10T1/2 cells. (A) Transfection efficiency was assessed via (A) fluorescence microscopy, (B) reverse transcription-quantitative PCR and (C) western blotting. (D) Immunofluorescence staining. Green indicates successfully transfected C3H10T1/2 cells, red indicates Ckip-1 expression in C3H10T1/2 cells and blue indicates nuclear staining. ${ }^{*} \mathrm{P}<0.05$. Ckip-1, casein kinase-2 interaction protein-1; sh, short hairpin RNA; Ctrl, control; EV, empty vector.

A

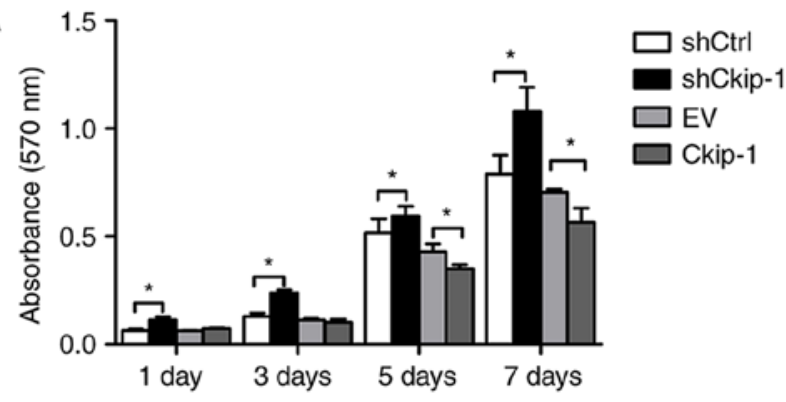

C

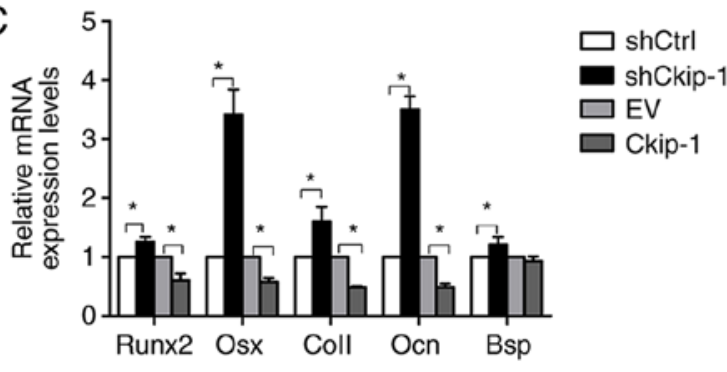

B
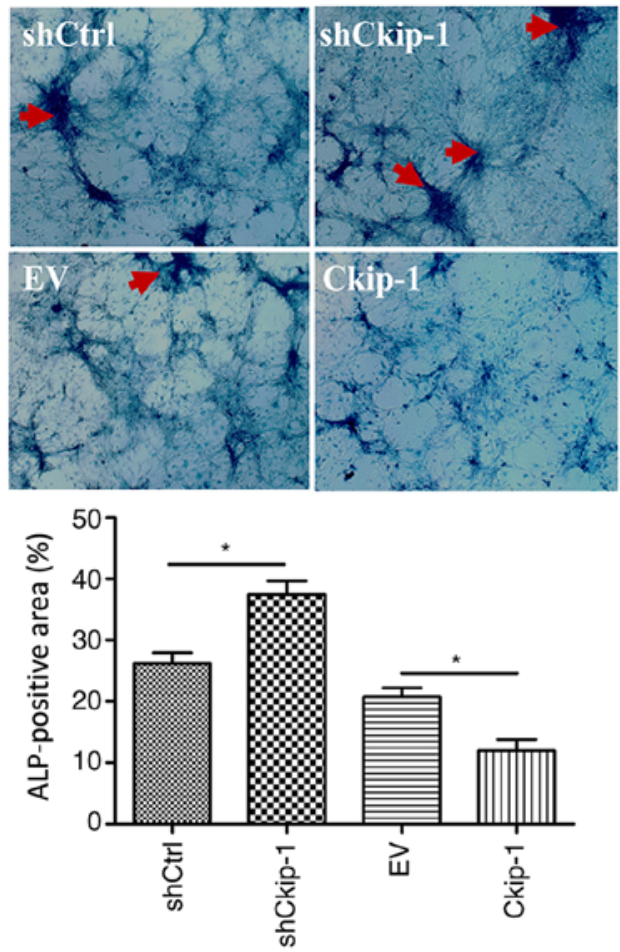

Figure 2. C3H10T1/2 cell proliferation and osteogenic differentiation. (A) Cell proliferation was assessed by performing an MTT assay on day 1,3, 5 and 7 . (B) Following 14-day osteoinduction, ALP activity was assessed via ALP staining (magnification, $\mathrm{x} 200$ ). Red arrows indicate the high positive black precipitate. (C) mRNA expression levels of five osteogenic makers (Runx2, Osx, Col1, Ocn and Bsp). "P<0.05. ALP, alkaline phosphatase; Runx2, RUNX family transcription factor 2; Osx, Osterix; Col1, type I collagen; Ocn, osteocalcin; Bsp, bone sialoprotein; sh, short hairpin RNA; Ctrl, control; EV, empty vector.

Ckip-1 knockdown and knockout increase the expression levels of Lrp5 in C3H1OT1/2 cells. The effects of Ckip-1 knockdown on the expression levels of Lrp5, Tcf1 and Lef1 in C3H10T1/2 cells in the $7 \mathrm{~d}$-shCkip-1 group were subsequently examined via RT-qPCR. The results indicated that the expression levels of Lrp5, Tcf1 and Lef1 were significantly increased in the $7 \mathrm{~d}$-shCkip- 1 group compared with the $7 \mathrm{~d}$-shCtrl group (Fig. 3A). The western blotting results indicated that the expression levels of Lrp5 were increased in the $7 \mathrm{~d}$-shCkip-1 group compared with the $7 \mathrm{~d}$-shCtrl group, whereas $\beta$-catenin expression levels were not altered. However, the expression levels of Lrp5 and $\beta$-catenin were markedly increased in the $14 \mathrm{~d}$-shCkip-1 group compared with the shCtrl group (Fig. 3B). To examine the cellular localization of Ckip-1 and Lrp5, immunofluorescence staining was performed (Fig. 3C). Colocalization of Ckip-1 (red) and 

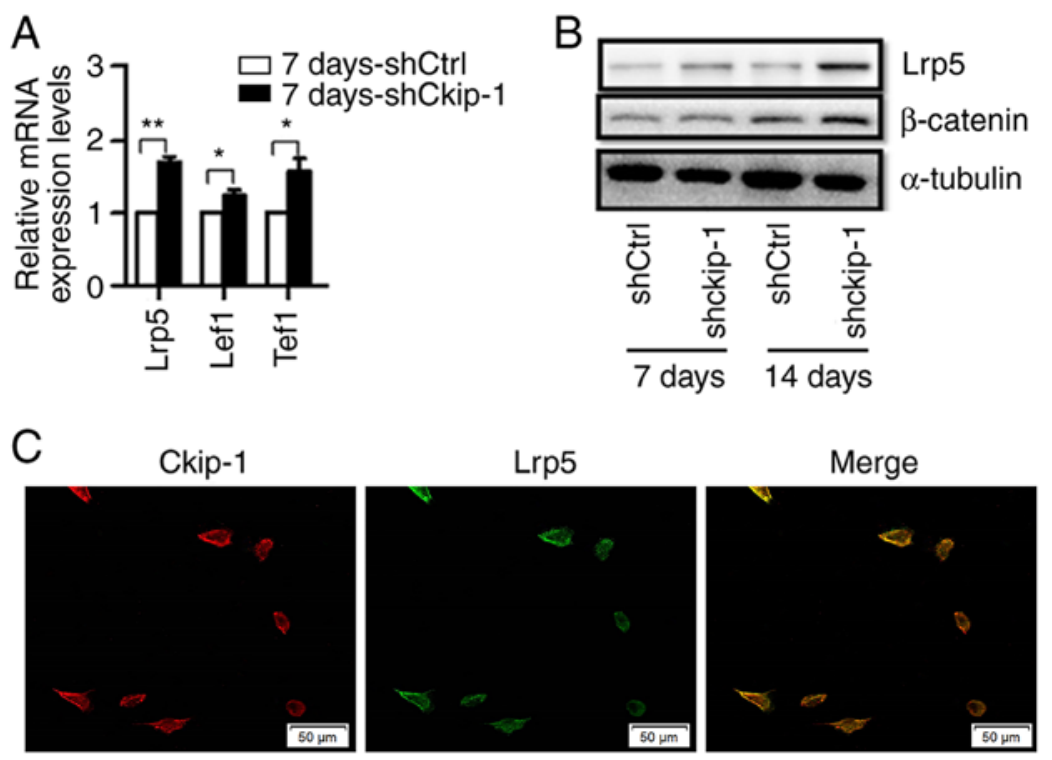

Figure 3. Expression of the key molecules of the Wnt/3-catenin signaling pathway in shCkip-1-transfected C3H10T1/2 cells. (A) Lrp5, Lef1, Tcf1 mRNA expression levels in shCkip-1-transfected C3H10T1/2 cells on day 7 of osteoinduction. (B) Protein expression levels of Lrp5 and $\beta$-catenin on day 7 and 14 of osteoinduction. (C) Co-localization of Lrp5 and Ckip-1 was assessed via immunofluorescence staining. Green represents Lrp5 staining and red indicates Ckip-1 staining. " $\mathrm{P}<0.05$ and ${ }^{* *} \mathrm{P}<0.01$. sh, short hairpin RNA; Ckip-1, casein kinase-2 interaction protein-1; Lrp5, LDL receptor related protein 5; Lef1, lymphoid enhancer binding factor 1 ; Tcf 1 , transcription factor 1 ; d, days.
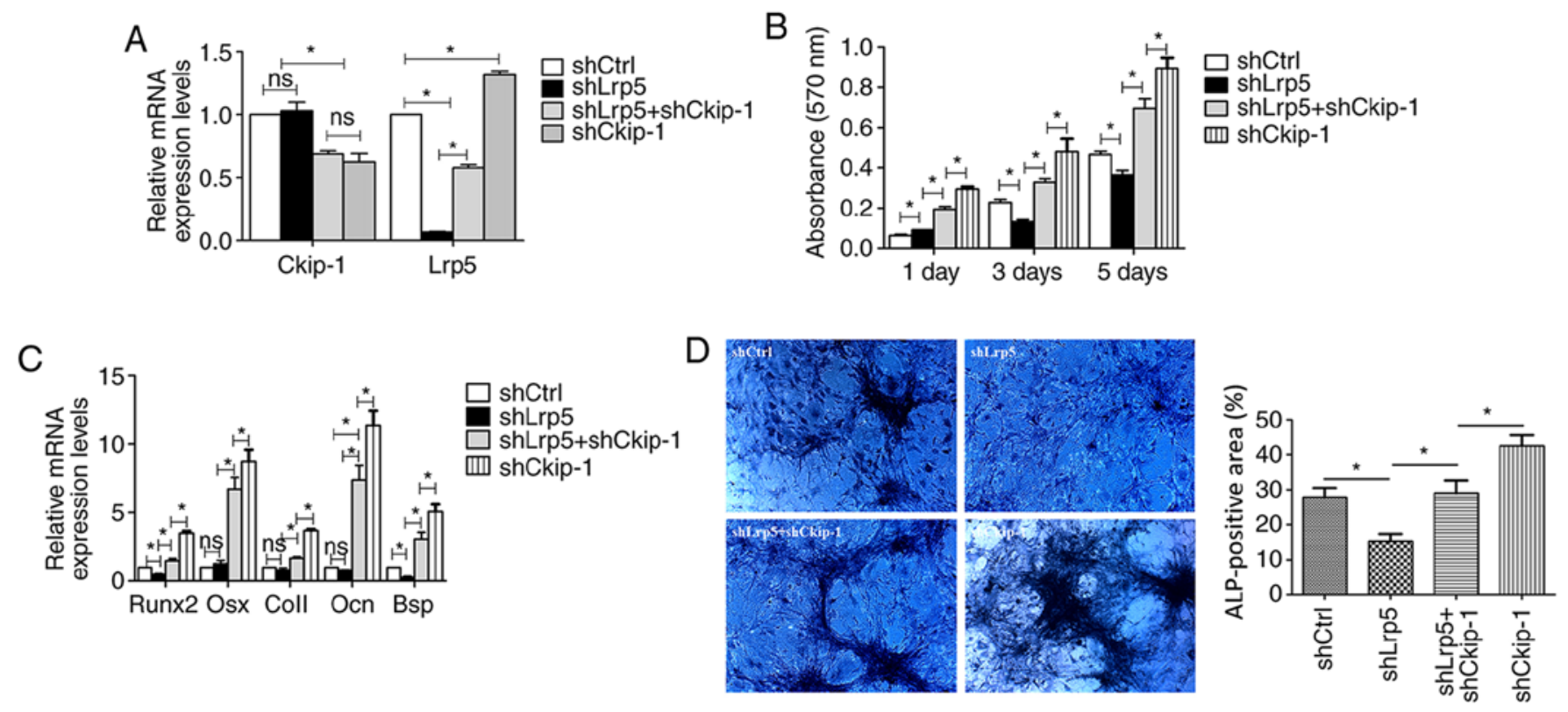

Figure 4. Rescue effect of shCkip-1 on shLrp5-transfected C3H10T1/2 cell proliferation and osteogenic differentiation. (A) Transfection efficiency of shLrp5 and shCkip-1. (B) Cell proliferation was assessed by performing an MTT assay on day 1,3 and 5. (C) mRNA expression levels of five osteogenic makers (Runx2, Osx, Col1, Ocn, Bsp). (D) ALP activity was assessed via ALP staining (magnification, x200). "P<0.05. sh, short hairpin RNA; Ckip-1, casein kinase-2 interaction protein-1; Lrp5, LDL receptor related protein 5; Runx2, RUNX family transcription factor 2; Osx, Osterix; Col1, type I collagen; Ocn, osteocalcin; Bsp, bone sialoprotein; ALP, alkaline phosphatase; Ctrl, control.

Lrp5 (green) was observed in C3H10T1/2 cells under normal conditions. Moreover, the results suggested that Ckip-1 highly accumulated in the vicinity of the membrane of C3H10T1/2 cells, which may imply an underlying membrane-associated role of Ckip-1.

Decreased cell proliferation in the Lrp5 knockdown group can be rescued by shCkip-1. Simultaneous Lrp5 and Ckip-1 knockdown was achieved by lentiviral transfection and confirmed via RT-qPCR. In accordance with the aforementioned results, the expression level of Lrp5 was significantly increased in the shCkip-1 and shLrp5 + shCkip-1 groups compared with shCtrl group (Fig. 4A). C3H10T1/2 cell proliferation and osteogenic differentiation were evaluated in the different groups. At 3 and 5 days, cell proliferation in the shLrp5 group was significantly reduced compared 
with the shCtrl group (Fig. 4B). At 1, 3 and 5 days in the shLrp5 + shCkip-1 group, cell proliferation was significantly higher compared with the shLrp5 group.

Involvement of Lrp5 in Ckip-1 knockdown-induced C3H10T1/2 cell osteogenic differentiation. The relative gene expression levels of osteogenic markers in the shLrp5, shCkip-1, shLrp5 + shCkip-1 and shCtrl groups were evaluated after 7-day osteoinduction. The majority of the osteogenic markers were expressed at lower levels in the shLrp5 group compared with the shCtrl group, with significantly decreased Runx2 and Bsp expression levels in the shLrp5 group compared with the shCtrl group (Fig. 4C). However, in the shLrp5 + shCkip-1 group, osteogenic-related marker expression levels were significantly increased compared with the shLrp5 group. Moreover, the expression levels of the osteogenic markers in the shCkip-1 group were significantly higher compared with the shLrp5 + shCkip-1 group. The ALP activity detection results further indicated the rescue response of shCkip-1 in C3H10T1/2 cell osteogenic differentiation following Lrp5 knockdown (Fig. 4D).

\section{Discussion}

The stimulation of MSC proliferation and osteogenic differentiation is a promising approach for the treatment of bone defects $(25,26)$. Ckip-1 can negatively regulate bone formation, and the canonical Wnt signaling pathway is one of the five osteogenesis signaling pathways $(8,27)$. In the present study, the role of Ckip-1 in the regulation of osteogenic differentiation via the canonical Wnt signaling pathway was assessed. Moreover, to the best of our knowledge, with the exception of the BMP signaling pathway (22), the association between the osteogenic signaling pathways and Ckip-1 has not been previous reported. In the present study, the common C3H10T1/2 MSC-like pluripotent cell line was used, which was obtained from mouse embryos and established by Reznikoff et al in 1973 (28). The effects of Ckip-1 on C3H10T1/2 cell proliferation and osteogenic differentiation were evaluated in the present study.

The results of the MTT assay indicated that C3H10T1/2 cell proliferation was enhanced and reduced in the shCkip-1 and Ckip-1 groups compared with the shCtrl and EV groups, respectively, indicating that Ckip-1 negatively regulated $\mathrm{C} 3 \mathrm{H} 10 \mathrm{~T} 1 / 2$ cell proliferation. ALP activity is upregulated during osteogenesis and is regarded as an early bone marker (29). The ALP staining and RT-qPCR assays suggested that Ckip-1 also negatively regulated C3H10T1/2 cell osteogenic differentiation.

Subsequently, the association between Ckip-1 and the canonical Wnt signaling pathway was investigated. Following immunofluorescence staining, the expression and localization of Ckip-1 were examined in the cell membrane, cytoplasm and nucleus under normal conditions. High accumulation of Ckip-1 was observed at the cell membrane, which indicated colocalization of Ckip-1 with the Lrp5 receptor. Previous studies have suggested that Ckip-1 is localized near the cell membrane and distributed throughout the cytoplasm or in the nucleus, suggesting that its function is determined by its cellular location (30-33). Therefore, it was hypothesized that membrane-associated Ckip-1 localization may account for the phenotype of the C3H10T1/2 cell line or for the expression of the membrane receptors.

Ckip-1 knockdown significantly increased the expression levels of the key molecules of the canonical Wnt signaling pathway (Lrp5, Lef1 and Tcf1) in C3H10T1/2 cells compared with the shCtrl group. Lrp5 knockdown was established in C3H10T1/2 cells, since the cell line exhibited high Lrp5 expression compared with the shCtrl group (Fig. 3A and B). Lrp5 is a transmembrane receptor that can bind to Wnt ligands and FZD proteins to initiate and activate the canonical Wnt signaling pathway (12). Related studies have demonstrated that disruption of Lrp5 can result in a low bone mass phenotype in postnatal mice, whereas Lrp5 knockdown can lead to a high bone mass phenotype $(34,35)$. The shCkip-1 group displayed increased expression levels of Lrp5 compared with the shCtrl group, as determined via RT-qPCR and western blotting. In addition, $\beta$-catenin is an important downstream molecule of the canonical Wnt signaling pathway $(36,37)$. Previous studies have indicated that deletion of $\beta$-catenin can negatively affect osteoblast differentiation and bone formation $(36,37)$. The western blotting results indicated that $\beta$-catenin expression levels were significantly increased on day 14 of osteoinduction in the shCkip-1 group compared with the shCtrl group, which was consistent with previous studies.

In the present study, the effect of Lrp5 knockdown on C3H10T1/2 cell proliferation and osteogenic differentiation was assessed. Moreover, the potential of Ckip-1 knockdown to reverse Lrp5 knockdown-mediated effects was also examined. Following lentiviral transfection, MTT and RT-qPCR assays were performed to evaluate cell proliferation and osteogenic marker expression, respectively. Cell proliferation was significantly decreased in the shLrp5 group on day 3 compared with the shCtrl group, indicating that Lrp5 may exert a potential positive effect on $\mathrm{C} 3 \mathrm{H} 10 \mathrm{~T} 1 / 2$ cell proliferation. The shLrp5 + shCkip-1 group displayed higher cell proliferation compared with the shLrp5 group, which implied a rescue role of shCkip-1. With regard to osteogenic differentiation, the expression levels of the osteogenic-associated genes of the shLrp5 + shCkip-1 group were significantly increased compared with the shCtrl and shLrp5 groups. The shLrp5 and shCtrl groups displayed no significant differences. However, the expression levels of almost all the genes investigated were slightly decreased in the shLrp5 group compared with the shCtrl group, which was consistent with previous findings demonstrating that Lrp5 may serve a positive regulatory role on bone formation $(34,35)$. Furthermore, it was hypothesized that the transmembrane receptor Lrp5 may serve as a potential target of Ckip-1 based on the membrane-associated colocalization of Ckip-1 and Lrp5 in C3H10T1/2 cells. However, further studies are required to verify the hypothesis, potentially by using a Wnt inhibitor.

In conclusion, the present study evaluated the effects of Ckip-1 on C3H10T1/2 cell proliferation and osteogenic differentiation, as well as the potential underlying mechanism. The results demonstrated that Ckip-1 negatively regulated C3H10T1/2 MSC cell proliferation and osteogenic differentiation via the canonical Wnt-signaling receptor Lrp5, which may provide a promising target for the improvement of BTE. 


\section{Acknowledgements}

Not applicable.

\section{Funding}

The present study was supported by the National Natural Science Foundation of China (grant no. 81670803).

\section{Availability of data and materials}

The datasets used and/or analyzed during the current study are available from the corresponding author on reasonable request.

\section{Authors' contributions}

YS and LK conceived the study. XH and JL designed the experiments. JL provided software. YG validated, formally analyzed and investigated the data. YH provided resources and performed experiments. XH wrote and reviewed the final manuscript. YS provided supervision. LK provided project administration and acquired funding. All authors read and approved the final manuscript.

\section{Ethics approval and consent to participate}

Not applicable.

\section{Patient consent for publication}

Not applicable.

\section{Competing interests}

The authors declare that they have no competing interests.

\section{References}

1. Li L, Lu H, Zhao Y, Luo J, Luo J, Yang L, Liu W and He Q Functionalized cell-free scaffolds for bone defect repair inspired by self-healing of bone fractures: A review and new perspectives. Mater Sci Eng C Mater Biol Appl 98: 1241-1251, 2019.

2. Liu M and Lv Y: Reconstructing bone with natural bone graft A review of in vivo studies in bone defect animal model. Nanomaterials (Basel) 8: E999, 2018.

3. Wei X, Liu B, Liu G, Yang F, Cao F, Dou X, Yu W, Wang B, Zheng G, Cheng L, et al: Mesenchymal stem cell-loaded porous tantalum integrated with biomimetic 3D collagen-based scaffold to repair large osteochondral defects in goats. Stem Cell Res Ther 10: 72, 2019.

4. Chen X, Fan H, Deng X, Wu L, Yi T, Gu L, Zhou C, Fan Y and Zhang X: Scaffold structural microenvironmental cues to guide tissue regeneration in bone tissue applications. Nanomaterials (Basel) 8: E960, 2018.

5. White KA and Olabisi RM: Spatiotemporal control strategies for bone formation through tissue engineering and regenerative medicine approaches. Adv Healthc Mater 8: e1801044, 2019.

6. El-Rashidy AA, Roether JA, Harhaus L, Kneser U and Boccaccini AR: Regenerating bone with bioactive glass scaffolds: A review of in vivo studies in bone defect models. Acta Biomater 62: 1-28, 2017.

7. Leyendecker Junior A,Gomes Pinheiro CC,LazzarettiFernandes T and Franco Bueno D: The use of human dental pulp stem cells for in vivo bone tissue engineering: A systematic review. J Tissue Eng 9: 2041731417752766, 2018.
8. Wehner D and Weidinger G: Signaling networks organizing regenerative growth of the zebrafish fin. Trends Genet 31: 336-343, 2015.

9. Ren L, Chen H, Song J, Chen X, Lin C, Zhang X, Hou N, Pan J, Zhou Z, Wang L, et al: MiR-454-3p-mediated Wnt/ $\beta$-catenin signaling antagonists suppression promotes breast cancer metastasis. Theranostics 9: 449-465, 2019.

10. Yuan K, Shamskhou EA, Orcholski ME, Nathan A, Reddy S, Honda H, Mani V, Zeng Y, Ozen MO, Wang L, et al: Loss of endothelial derived WNT5A is associated with reduced pericyte recruitment and small vessel loss in pulmonary arterial hypertension. Circulation 139: 1710-1724, 2019.

11. Gong B, Shen W, Xiao W, Meng Y, Meng A and Jia S: The Sec14-like phosphatidylinositol transfer proteins Sec1413/SEC14L2 act as GTPase proteins to mediate Wnt/Ca ${ }^{2+}$ signaling. Elife 6: e26362, 2017.

12. Baron R and Kneissel M: WNT signaling in bone homeostasis and disease: From human mutations to treatments. Nat Med 19: 179-192, 2013.

13. Taipaleenmäki H, Abdallah BM, Aldahmash A, Säämänen AM and Kassem M: Wnt signalling mediates the cross-talk between bone marrow derived pre-adipocytic and pre-osteoblastic cell populations. Exp Cell Res 317: 745-756, 2011.

14. Day TF, Guo X, Garrett-Beal L and Yang Y: Wnt/beta-catenin signaling in mesenchymal progenitors controls osteoblast and chondrocyte differentiation during vertebrate skeletogenesis. Dev Cell 8: 739-750, 2005.

15. D'Alimonte I, Lannutti A, Pipino C, Di Tomo P, Pierdomenico L, Cianci E, Antonucci I, Marchisio M, Romano M, Stuppia L, et al: Wnt signaling behaves as a 'master regulator' in the osteogenic and adipogenic commitment of human amniotic fluid mesenchymal stem cells. Stem Cell Rev Rep 9: 642-654, 2013.

16. Zuo R, Liu M, Wang Y, Li J, Wang W, Wu J, Sun C, Li B, Wang Z, Lan W, et al: BM-MSC-derived exosomes alleviate radiation-induced bone loss by restoring the function of recipient BM-MSCs and activating Wnt $/ \beta$-catenin signaling. Stem Cell Res Ther 10: 30, 2019.

17. Fan J, An X, Yang Y, Xu H, Fan L, Deng L, Li T, Weng X, Zhang J and Zhao RC: MiR-1292 targets FZD4 to regulate senescence and osteogenic differentiation of stem cells in TE/SJ/mesenchymal tissue system via the Wnt/ $\beta$-catenin pathway. Aging Dis 9: 1103-1121, 2018.

18. Williams BO: LRP5: From bedside to bench to bone. Bone 102: 26-30, 2017.

19. Li T, Li H, Wang Y, Li T, Fan J, Xiao K, Zhao RC and Weng X: microRNA-23a inhibits osteogenic differentiation of human bone marrow-derived mesenchymal stem cells by targeting LRP5. Int J Biochem Cell Biol 72: 55-62, 2016.

20. Borrell-Pagès M, Romero JC and Badimon L: LRP5 deficiency down-regulates Wnt signalling and promotes aortic lipid infiltration in hypercholesterolaemic mice. J Cell Mol Med 19: 770-777, 2015.

21. Semenov MV and He X: LRP5 mutations linked to high bone mass diseases cause reduced LRP5 binding and inhibition by SOST. J Biol Chem 281: 38276-38284, 2006.

22. Nie J, Liu L, He F, Fu X, Han W and Zhang L: CKIP-1: A scaffold protein and potential therapeutic target integrating multiple signaling pathways and physiological functions. Ageing Res Rev 12: 276-281, 2013.

23. Lu K, Yin X, Weng T, Xi S, Li L, Xing G, Cheng X, Yang X, Zhang $\mathrm{L}$ and $\mathrm{He} \mathrm{F}$ : Targeting WW domains linker of HECT-type ubiquitin ligase Smurf1 for activation by CKIP-1. Nat Cell Biol 10: 994-1002, 2008.

24. Livak KJ and Schmittgen TD: Analysis of relative gene expression data using real-time quantitative PCR and the 2(-Delta Delta C(T)) method. Methods 25: 402-408, 2001.

25. Chen BY, Wang X, Chen LW and Luo ZJ: Molecular targeting regulation of proliferation and differentiation of the bone marrow-derived mesenchymal stem cells or mesenchymal stromal cells. Curr Drug Targets 13: 561-571, 2012.

26. Lee DJ, Kwon J, Current L, Yoon K, Zalal R, Hu X, Xue P and Ko CC: Osteogenic potential of mesenchymal stem cells from rat mandible to regenerate critical sized calvarial defect. J Tissue Eng 10: 2041731419830427, 2019.

27. Moore ER and Jacobs CR: The primary cilium as a signaling nexus for growth plate function and subsequent skeletal development. J Orthop Res 36: 533-545, 2018.

28. Reznikoff CA, Brankow DW and Heidelberger C: Establishment and characterization of a cloned line of $\mathrm{C} 3 \mathrm{H}$ mouse embryo cells sensitive to postconfluence inhibition of division. Cancer Res 33: 3231-3238, 1973 . 
29. Hashimi SM: Exogenous noggin binds the BMP-2 receptor and induces alkaline phosphatase activity in osteoblasts. J Cell Biochem 120: 13237-13242, 2019.

30. Li D, Zhu H, Liang C, Li W, Xing G, Ma L, Ding L, Zhang Y, $\mathrm{He} F$ and Zhang L: CKIP-1 suppresses the adipogenesis of mesenchymal stem cells by enhancing HDAC1-associated repression of C/EBPa. J Mol Cell Biol 6: 368-379, 2014.

31. Safi A, Vandromme M, Caussanel S, Valdacci L, Baas D, Vidal M, Brun G, Schaeffer L and Goillot E: Role for the pleckstrin homology domain-containing protein CKIP-1 in phosphatidylinositol 3-kinase-regulated muscle differentiation. Mol Cell Biol 24: 1245-1255, 2004.

32. Zhang L, Xing G, Tie Y, Tang Y, Tian C, Li L, Sun L, Wei H, Zhu Y and He F: Role for the pleckstrin homology domain-containing protein CKIP-1 in AP-1 regulation and apoptosis. EMBO J 24: 766-778, 2005.

33. Bosc DG, Graham KC, Saulnier RB, Zhang C, Prober D, Gietz RD and Litchfield DW: Identification and characterization of CKIP-1, a novel pleckstrin homology domain-containing protein that interacts with protein kinase CK2. J Biol Chem 275: 14295-14306, 2000
34. Kato M, Patel MS, Levasseur R, Lobov I, Chang BHJ Glass DA II, Hartmann C, Li L, Hwang TH, Brayton CF, et al: Cbfal-independent decrease in osteoblast proliferation, osteopenia, and persistent embryonic eye vascularization in mice deficient in Lrp5, a Wnt coreceptor. J Cell Biol 157: 303-314, 2002.

35. Cui Y, Niziolek PJ, MacDonald BT, Zylstra CR, Alenina N, Robinson DR, Zhong Z, Matthes S, Jacobsen CM, Conlon RA, et al: Lrp5 functions in bone to regulate bone mass. Nat Med 17: 684-691, 2011.

36. Hu H, Hilton MJ, Tu X, Yu K, Ornitz DM and Long F: Sequential roles of Hedgehog and Wnt signaling in osteoblast development. Development 132: 49-60, 2005.

37. Hill TP, Später D, Taketo MM, Birchmeier W and Hartmann C: Canonical Wnt/beta-catenin signaling prevents osteoblasts from differentiating into chondrocytes. Dev Cell 8: 727-738, 2005.

(i) (9) This work is licensed under a Creative Common

EY NG NO Attribution-NonCommercial-NoDerivatives 4.0 International (CC BY-NC-ND 4.0) License. 